\title{
DENSE HOLOMORPHIC CURVES IN SPACES OF HOLOMORPHIC MAPS AND APPLICATIONS TO UNIVERSAL MAPS
}

\author{
YUTA KUSAKABE
}

\begin{abstract}
We study when there exists a dense holomorphic curve in a space of holomorphic maps from a Stein space. We first show that for any bounded convex domain $\Omega \Subset \mathbb{C}^{n}$ and any connected complex manifold $Y$, the space $\mathcal{O}(\Omega, Y)$ contains a dense holomorphic disc. Our second result states that $Y$ is an Oka manifold if and only if for any Stein space $X$ there exists a dense entire curve in every path component of $\mathcal{O}(X, Y)$.

In the second half of this paper, we apply the above results to the theory of universal functions. It is proved that for any bounded convex domain $\Omega \Subset \mathbb{C}^{n}$, any fixed-point-free automorphism of $\Omega$ and any connected complex manifold $Y$, there exists a universal map $\Omega \rightarrow Y$. We also characterize Oka manifolds by the existence of universal maps.
\end{abstract}

\section{INTRODUCTION}

Let $Y$ be a complex space (throughout this paper always taken to be reduced and second countable). It is a fundamental problem whether a given complex space $Z$ admits a holomorphic map $Z \rightarrow Y$ with dense image. In this paper, we study the following more general problem for a Stein space $X$ : When does there exist a dense holomorphic map from $Z$ to a subspace $\mathcal{S}$ of the space $\mathcal{O}(X, Y)$ of holomorphic maps from $X$ to $Y$ equipped with the compact-open topology? Here we say a map $f: Z \rightarrow \mathcal{S}$ to be holomorphic if the associated map $Z \times X \rightarrow Y,(z, x) \mapsto f(z)(x)$ is holomorphic in the usual sense. Let us introduce the following terminology.

Definition 1.1. A subspace $\mathcal{S} \subset \mathcal{O}(X, Y)$ is said to be $Z$-dominated if there exists a holomorphic map $Z \rightarrow \mathcal{S}$ with dense image.

Let us review the case when $X$ is a singleton, i.e. $\mathcal{O}(X, Y) \cong Y$. Winkelmann [10] proved that for any irreducible complex space $Z$ admitting a nonconstant bounded holomorphic function, the unit disc $\mathbb{D}$ is $Z$-dominated. Correspondingly, Chen and Wang [3] proved that for any irreducible complex space $Z$ admitting a nonconstant holomorphic function, the complex line $\mathbb{C}$ is $Z$-dominated. Since the composition of two dense holomorphic maps has dense image, our problem

2010 Mathematics Subject Classification. 32E10, 32H02, 54C35, 30K20, 47A16.

Key words and phrases. space of holomorphic maps, Stein space, Oka manifold, universal function, composition operator. 
reduces to the $\mathbb{D}$-dominability or the $\mathbb{C}$-dominability of a subspace $\mathcal{S} \subset \mathcal{O}(X, Y)$ in most cases even if $X$ is not a singleton. For this reason, we study only the $\mathbb{D}$-dominability and the $\mathbb{C}$-dominability.

In his paper [10], Winkelmann also proved that any irreducible complex space is $\mathbb{D}$-dominated. Our first main result is the following partial generalization to spaces of holomorphic maps. It is proved in Section 2 .

Theorem 1.2. Let $\Omega \Subset \mathbb{C}^{n}$ be a bounded convex domain and $Y$ a connected complex manifold. Then $\mathcal{O}(\Omega, Y)$ is $\mathbb{D}$-dominated.

If we replace $\Omega$ by a more general pseudoconvex domain or replace $Y$ by a singular space, then this theorem is no longer true (see examples at the end of Section 21). This is due to the reducibility with respect to the Zariski topologies on the spaces of holomorphic maps (see Definition 2.6).

In Section 3, we study the $\mathbb{C}$-dominability. A connected Oka manifold (see Definition (3.1) is a typical example of a $\mathbb{C}$-dominated space, but the converse is not true. For example, for a discrete sequence $D \subset \mathbb{C}^{2}$ which is unavoidable by nondegenerate holomorphic self-maps of $\mathbb{C}^{2}$ (cf. [4, §4.7]), the complement $\mathbb{C}^{2} \backslash D$ is $\mathbb{C}$-dominated for dimensional reasons, but it is not Oka. By the following second main result, however, a complex manifold $Y$ must be Oka if we further assume that every space of holomorphic maps to $Y$ is $\mathbb{C}$-dominated.

Theorem 1.3. For a connected complex manifold $Y$, the following are equivalent:

(1) $Y$ is Oka.

(2) For any Stein space $X$, every path component $\mathcal{P} \subset \mathcal{O}(X, Y)$ is $\mathbb{C}$-dominated.

(3) For any bounded convex domain $\Omega \Subset \mathbb{C}^{n}$, the space $\mathcal{O}(\Omega, Y)$ is $\mathbb{C}$-dominated.

In Section 4, we give applications of the above results to the theory of universal functions. In 1929, Birkhoff constructed for each $a \in \mathbb{C} \backslash\{0\}$ an entire function $F(z) \in \mathcal{O}(\mathbb{C})$ such that $\{F(z+j a)\}_{j \in \mathbb{N}}$ is dense in $\mathcal{O}(\mathbb{C})($ cf. [7]). Such a function is called a universal function. Universal functions $X \rightarrow \mathbb{C}$ on more general spaces $X$ have been studied intensively. In this paper, we make the first attempt to develop the theory of universal maps $X \rightarrow Y$ to more general targets $Y$.

Definition 1.4. Let $\tau \in$ Aut $X$ be an automorphism and assume that $\mathcal{S} \subset$ $\mathcal{O}(X, Y)$ is a $\tau^{*}$-invariant subspace, i.e. $\tau^{*} \mathcal{S} \subset \mathcal{S}$, where $\tau^{*}: \mathcal{O}(X, Y) \rightarrow \mathcal{O}(X, Y)$ is the precomposition map. A holomorphic map $F \in \mathcal{S}$ is called an $\mathcal{S}$-universal map for $\tau$ if $\left\{F \circ \tau^{j}\right\}_{j \in \mathbb{N}}$ is dense in $\mathcal{S}$.

More recently, Zając [1] characterized automorphisms admitting universal functions, or universal maps to $\mathbb{C}$. Andrist and Wold [2] call such automorphisms generalized translations (see Definition 4.3). It is natural to consider universal maps for generalized translations. For a bounded convex domain, a generalized translation is just a fixed-point-free automorphism (see Proposition 4.7). The following is proved as an application of Theorem 1.2 . 
Theorem 1.5. Let $\Omega \Subset \mathbb{C}^{n}$ be a bounded convex domain, $\tau \in$ Aut $\Omega$ a generalized translation and $Y$ a connected complex manifold. Then there exists an $\mathcal{O}(\Omega, Y)$ universal map for $\tau$.

For a universal map from a more general Stein space, we have the following result which is an application of Theorem 1.3,

Theorem 1.6. For a connected complex manifold $Y$, the following are equivalent:

(1) $Y$ is Oka.

(2) For any Stein space $X$, any generalized translation $\tau \in$ Aut $X$ and any $\tau^{*}$ invariant path component $\mathcal{P} \subset \mathcal{O}(X, Y)$, there exists a $\mathcal{P}$-universal map.

(3) For any convex domain $\Omega \subset \mathbb{C}^{n}$ and any generalized translation $\tau \in$ Aut $\Omega$, there exists an $\mathcal{O}(\Omega, Y)$-universal map.

We also have an application of universal maps. Forstnerič and Winkelmann [6] proved that the set of dense holomorphic discs in a connected complex manifold is dense in the space of holomorphic discs. By Theorem 1.5, we may easily obtain the following generalization to several variables because a universal map has dense image and a generic map is universal if there exists some universal map.

Corollary 1.7. For any connected complex manifold $Y$, the set of dense holomorphic balls $\mathbb{B}^{n} \rightarrow Y$ is dense in $\mathcal{O}\left(\mathbb{B}^{n}, Y\right)$. It holds also for a polydisc $\mathbb{D}^{n}$.

Finally, let us give a few remarks and notations which will be used in this paper.

Remark 1.8. Let $X, Y$ and $Z$ be complex spaces.

(1) The space $\mathcal{O}(X, Y)$ of holomorphic maps is second countable and completely metrizable. In particular, it is a separable Baire space. We omit the proof but it follows from the assumption that $X$ and $Y$ are second countable.

(2) We denote by $\mathcal{O}(X, \mathcal{O}(Y, Z))$ the space of holomorphic maps $X \rightarrow \mathcal{O}(Y, Z)$. This space is naturally homeomorphic to $\mathcal{O}(X \times Y, Z)$ by definition.

(3) For a holomorphic map $f: X \rightarrow Y$, we denote by $f^{*}: \mathcal{O}(Y, Z) \rightarrow \mathcal{O}(X, Z)$ and $f_{*}: \mathcal{O}(Z, X) \rightarrow \mathcal{O}(Z, Y)$ the precomposition map and the postcomposition map, respectively. We denote by ev $: \mathcal{O}(X, Y) \times X \rightarrow Y$ the evaluation map $(g, x) \mapsto$ $g(x)$. Note that these maps define the postcomposition maps $\mathcal{O}(S, \mathcal{O}(Y, Z)) \rightarrow$ $\mathcal{O}(S, \mathcal{O}(X, Z)), \mathcal{O}(S, \mathcal{O}(Z, X)) \rightarrow \mathcal{O}(S, \mathcal{O}(Z, Y))$ and $\mathcal{O}(S, \mathcal{O}(X, Y)) \times \mathcal{O}(S, X) \rightarrow$ $\mathcal{O}(S, Y)$ between spaces of holomorphic maps for any complex space $S$.

\section{The $\mathbb{D}$-Dominability: Proof of Theorem 1.2}

Our proof of Theorem 1.2 is based on the ideas of Forstnerič and Winkelmann [6]. We shall need two lemmas.

Lemma 2.1. Assume that $\Omega \Subset \mathbb{C}^{n}$ is a bounded convex domain and $Y$ is a connected complex manifold. Let $f: \mathbb{D} \rightarrow \mathcal{O}(\Omega, Y)$ be a holomorphic disc and $\mathcal{U} \subset \mathcal{O}(\Omega, Y)$ a nonempty open subset. Then there exists a sequence $\left\{f_{j}: W_{j} \rightarrow\right.$ 
$\mathcal{O}(\Omega, Y)\}_{j \in \mathbb{N}}$ of holomorphic maps from open neighborhoods of $\overline{\mathbb{D}} \cup[1,2] \subset \mathbb{C}$ such that

(1) $\left.f_{j}\right|_{\mathbb{D}} \rightarrow f$ as $j \rightarrow \infty$, and

(2) $f_{j}(2) \in \mathcal{U}$ for all $j \in \mathbb{N}$.

To prove this lemma, we need the following special case of Theorem 3.7.2 in [4]. Note that for any compact polynomially convex subset $0 \in L \subset \mathbb{C}^{n}$ the subset $((\overline{\mathbb{D}} \cup\{2\}) \times L) \cup([1,2] \times\{0\}) \subset \mathbb{C}^{n+1}$ is also polynomially convex.

Proposition 2.2. Assume that $0 \in \Omega \Subset \mathbb{C}^{n}$ is a bounded convex domain and $Y$ is a complex manifold. Set $K=(\overline{\mathbb{D}} \cup\{2\}) \times \bar{\Omega}$ and $I=[1,2] \times\{0\}$. Let $W \subset \mathbb{C}^{n+1}$ be an open neighborhood of $K$ and $f: W \cup I \rightarrow Y$ a continuous map which is holomorphic on $W$. Then there exists a sequence $\left\{f_{j}: W_{j} \rightarrow Y\right\}_{j \in \mathbb{N}}$ of holomorphic maps from open neighborhoods of $K \cup I \subset \mathbb{C}^{n+1}$ such that

(1) $\left.\left.f_{j}\right|_{K \cup I} \rightarrow f\right|_{K \cup I}$ as $j \rightarrow \infty$, and

(2) $\left.f_{j}(2, \cdot)\right|_{\Omega}=\left.f(2, \cdot)\right|_{\Omega}$ for all $j \in \mathbb{N}$.

For $a \in \mathbb{C}$ and $S \subset \mathbb{C}^{n}$, we write $a S=\{a z: z \in S\} \subset \mathbb{C}^{n}$.

Proof of Lemma 2.1. We may assume that $\Omega$ contains the origin $0 \in \mathbb{C}^{n}$. Let us consider the associated holomorphic map $\widehat{f}: \mathbb{D} \times \Omega \rightarrow Y,(z, x) \mapsto f(z)(x)$. For each $r>1$, the map $(z, x) \mapsto \widehat{f}\left(r^{-1} z, r^{-1} x\right)$ is holomorphic on the neighborhood $r(\mathbb{D} \times \Omega)$ of $\overline{\mathbb{D}} \times \bar{\Omega}$ and converges to $\widehat{f}$ on $\mathbb{D} \times \Omega$ as $r \searrow 1$. Therefore, we may assume that $\widehat{f}$ is holomorphic on a neighborhood of $\overline{\mathbb{D}} \times \bar{\Omega}$ from the beginning. Take a holomorphic map $u \in \mathcal{U}$. For the same reason, we may assume that $u$ is holomorphic on a neighborhood of $\bar{\Omega}$.

Since $Y$ is connected, we may easily find an open neighborhood $V \subset \mathbb{C}^{n+1}$ of $(\overline{\mathbb{D}} \cup\{2\}) \times \bar{\Omega}$ and a continuous map $g: V \cup([1,2] \times\{0\}) \rightarrow Y$ such that $\left.g\right|_{V}$ is holomorphic, $\left.g\right|_{\mathbb{D} \times \Omega}=\widehat{f}$ and $\left.g(2, \cdot)\right|_{\Omega}=u$. By Proposition 2.2. there exists a sequence $\left\{g_{j}: V_{j} \rightarrow Y\right\}_{j \in \mathbb{N}}$ of holomorphic maps from open neighborhoods of $((\overline{\mathbb{D}} \cup\{2\}) \times \bar{\Omega}) \cup([1,2] \times\{0\}) \subset \mathbb{C}^{n+1}$ such that

(a) $\left.g_{j}\right|_{\mathbb{D} \times \Omega} \rightarrow \widehat{f}$ as $j \rightarrow \infty$, and

(b) $\left.g_{j}(2, \cdot)\right|_{\Omega}=u$ for all $j \in \mathbb{N}$.

For each fixed $j \in \mathbb{N}$, choose open sets $\overline{\mathbb{D}} \cup\{2\} \subset V_{\overline{\mathbb{D}} \cup\{2\}} \subset \mathbb{C}, \bar{\Omega} \subset V_{\bar{\Omega}} \subset$ $\mathbb{C}^{n},[1,2] \subset V_{[1,2]} \subset \mathbb{C}$ and $0 \in V_{0} \subset \mathbb{C}^{n}$ such that $\left(V_{\overline{\mathbb{D}} \cup\{2\}} \times V_{\bar{\Omega}}\right) \cup\left(V_{[1,2]} \times V_{0}\right) \subset V_{j}$. Since $t \bar{\Omega} \subset V_{\bar{\Omega}}$ for all $t \in[0,1]$, there exists an open neighborhood $N \subset \mathbb{C}$ of $[0,1]$ such that $z \Omega \subset V_{\bar{\Omega}}$ for all $z \in N$. Choose a small number $\varepsilon>0$ such that $z \Omega \subset V_{0}$ for all $z \in \varepsilon \mathbb{D}$. Note that there exists a continuous function $\chi$ on a neighborhood of $\overline{\mathbb{D}} \cup[1,2]$ with values in $[0,1] \subset N$ such that $\chi \equiv 1$ on a neighborhood of $\overline{\mathbb{D}} \cup\{2\}$ and $\chi \equiv 0$ on $[1,2] \backslash V_{\overline{\mathbb{D}} \cup\{2\}}$. Again by Proposition 2.2, there exists a holomorphic map $\rho_{j}: W_{j} \rightarrow N$ from an open neighborhood $W_{j} \subset V_{\overline{\mathbb{D}} \cup\{2\}} \cup V_{[1,2]}$ of $\overline{\mathbb{D}} \cup[1,2]$ such that 
(i) $\left.\rho_{j}\right|_{\mathbb{D}}$ is (arbitrarily) close to the constant map 1 ,

(ii) $\left|\rho_{j}(z)\right|<\varepsilon$ for all $z \in[1,2] \backslash V_{\overline{\mathbb{D}} \cup\{2\}}$, and

(iii) $\rho_{j}(2)=1$.

Shrinking $W_{j}$ if necessary, we may assume that $\left|\rho_{j}(z)\right|<\varepsilon$ for all $z \in W_{j} \backslash V_{\overline{\mathbb{D}} \cup\{2\}}$. By our construction, the map $(z, x) \mapsto g_{j}\left(z, \rho_{j}(z) x\right)$ is defined and holomorphic on $W_{j} \times \Omega$. We denote this map by $\widehat{f}_{j}: W_{j} \times \Omega \rightarrow Y$.

By the properties (a) and (i), choosing $\left\{\rho_{j}\right\}_{j \in \mathbb{N}}$ suitably, we may assume that $\left.\left.\widehat{f}_{j}\right|_{\mathbb{D} \times \Omega} \rightarrow \widehat{f}\right|_{\mathbb{D} \times \Omega}$ as $j \rightarrow \infty$. The properties (b) and (iii) implies that $\left.\widehat{f}_{j}(2, \cdot)\right|_{\Omega}=$ $u \in \mathcal{U}$ for all $j \in \mathbb{N}$. Therefore, the associated maps $f_{j}: W_{j} \rightarrow \mathcal{O}(\Omega, Y)$ to $\widehat{f}_{j}$ have the required properties.

The following lemma is an immediate consequence of Lemma 1 in [6].

Lemma 2.3. Let $\left\{W_{j}\right\}_{j \in \mathbb{N}}$ be a sequence of open neighborhoods of $\overline{\mathbb{D}} \cup[1,2] \subset \mathbb{C}$. Then there exists a sequence $\left\{\varphi_{j}: \mathbb{D} \rightarrow W_{j}\right\}_{j \in \mathbb{N}}$ of holomorphic discs such that

(1) $\varphi_{j} \rightarrow \operatorname{id}_{\mathbb{D}}$ as $j \rightarrow \infty$, and

(2) $2 \in \varphi_{j}(\mathbb{D})$ for all $j \in \mathbb{N}$.

Proof of Theorem 1.2. Choose a countable base $\left\{\mathcal{U}_{j}\right\}_{j \in \mathbb{N}}$ for the topology of $\mathcal{O}(\Omega, Y)$ and assume that $\mathcal{U}_{j} \neq \emptyset$ for all $j \in \mathbb{N}$. Let us consider the open subsets $\mathcal{V}_{j}=\{f \in$ $\left.\mathcal{O}(\mathbb{D}, \mathcal{O}(\Omega, Y)): f(\mathbb{D}) \cap \mathcal{U}_{j} \neq \emptyset\right\}$ of $\mathcal{O}(\mathbb{D}, \mathcal{O}(\Omega, Y))$. Note that $\bigcap_{j \in \mathbb{N}} \mathcal{V}_{j}$ is the set of dense holomorphic discs $\mathbb{D} \rightarrow \mathcal{O}(\Omega, Y)$. It suffices to show that each $\mathcal{V}_{j}$ is dense in $\mathcal{O}(\mathbb{D}, \mathcal{O}(\Omega, Y))$ because $\mathcal{O}(\mathbb{D}, \mathcal{O}(\Omega, Y))$ is a Baire space (see Remark 1.8).

For any holomorphic disc $f: \mathbb{D} \rightarrow \mathcal{O}(\Omega, Y)$, by Lemma 2.1, there exists a sequence $\left\{f_{k}: W_{k} \rightarrow \mathcal{O}(\Omega, Y)\right\}_{k \in \mathbb{N}}$ of holomorphic maps from open neighborhoods of $\overline{\mathbb{D}} \cup[1,2]$ such that $\left.f_{k}\right|_{\mathbb{D}} \rightarrow f$ as $k \rightarrow \infty$ and $f_{k}(2) \in \mathcal{U}_{j}$ for all $k \in \mathbb{N}$. By Lemma [2.3, there exists a sequence $\left\{\varphi_{k}: \mathbb{D} \rightarrow W_{k}\right\}_{k \in \mathbb{N}}$ of holomorphic discs such that $f_{k} \circ \varphi_{k} \rightarrow f$ as $k \rightarrow \infty$ and $f_{k} \circ \varphi_{k}(\mathbb{D}) \cap \mathcal{U}_{j} \neq \emptyset$ for all $k \in \mathbb{N}$. This proves that $\mathcal{V}_{j}$ is dense in $\mathcal{O}(\mathbb{D}, \mathcal{O}(\Omega, Y))$.

Remark 2.4. The above proof also shows that for a connected complex manifold $Y$ the set of dense holomorphic discs $\mathbb{D} \rightarrow \mathcal{O}(\Omega, Y)$ is dense in $\mathcal{O}(\mathbb{D}, \mathcal{O}(\Omega, Y))$. In fact, if $\mathcal{O}(\mathbb{D} \times \Omega, Y)$ is $\mathbb{D}$-dominated for a complex space $Y$, the set of dense holomorphic $\operatorname{discs} \mathbb{D} \rightarrow \mathcal{O}(\Omega, Y)$ must be dense. This can be seen as follows. By Corollary 4.9, the $\mathbb{D}$-dominability of $\mathcal{O}(\mathbb{D} \times \Omega, Y)$ implies that there exists an $\mathcal{O}(\mathbb{D} \times \Omega, Y)$ universal map for a generalized translation of the form $\tau=\sigma \times \operatorname{id}_{\Omega}$. This gives an $\mathcal{O}(\mathbb{D}, \mathcal{O}(\Omega, Y)$ )-universal map for $\sigma$ (in the obvious sense). Then it follows that a generic holomorphic disc $\mathbb{D} \rightarrow \mathcal{O}(\Omega, Y)$ is an $\mathcal{O}(\mathbb{D}, \mathcal{O}(\Omega, Y)$ )-universal map and has dense image.

In fact, we may obtain the following more precise result by the arguments in [6] and the above lemmas with minor modifications, but we omit its proof. We denote by $\left.\mathcal{O}(\bar{\Omega}, Y)\right|_{\Omega}$ the set of holomorphic maps $\Omega \rightarrow Y$ which can be extended to open neighborhoods of $\bar{\Omega}$. 
Proposition 2.5. Let $\Omega \Subset \mathbb{C}^{n}$ be a bounded convex domain and $Y$ a connected complex manifold. Then for every countable subset $\left.\mathcal{S} \subset \mathcal{O}(\bar{\Omega}, Y)\right|_{\Omega}$, the set $\{f \in$ $\mathcal{O}(\mathbb{D}, \mathcal{O}(\Omega, Y)): \mathcal{S} \subset f(\mathbb{D})\}$ is dense in $\mathcal{O}(\mathbb{D}, \mathcal{O}(\Omega, Y))$.

Note that we cannot omit the assumption $\left.\mathcal{S} \subset \mathcal{O}(\bar{\Omega}, Y)\right|_{\Omega}$ because bounded convex domains are immobile. Recall that a complex space $Y$ is said to be immobile if every holomorphic map $f: \mathbb{D} \times Y \rightarrow Y$ such that $f(0, y)=y$ for all $y \in Y$ satisfies $f(z, y)=y$ for all $(z, y) \in \mathbb{D} \times Y$. It is equivalent to saying that every nonconstant holomorphic disc $\mathbb{D} \rightarrow \mathcal{O}(Y, Y)$ does not intersect Aut $Y$. It is known that every Kobayashi hyperbolic space is immobile (cf. [8, Theorem 5.4.5]).

We end this section with some examples of spaces of holomorphic maps which are not $\mathbb{D}$-dominated. Let us introduce the notion of a Zariski topology on a space of holomorphic maps because these examples can be explained by the reducibility with respect to their Zariski topologies. This notion is also used in Section 4 .

Definition 2.6. We say a closed subset $\mathcal{S} \subset \mathcal{O}(X, Y)$ to be Zariski closed if for any holomorphic map $f: Z \rightarrow \mathcal{O}(X, Y)$ from any complex space $Z$, the preimage $f^{-1}(\mathcal{S}) \subset Z$ is Zariski closed. Zariski closed subsets define the Zariski topology on $\mathcal{O}(X, Y)$.

Note that a holomorphic map $f: Z \rightarrow \mathcal{O}(X, Y)$ is continuous with respect to the Zariski topology. Thus, if $Z$ is irreducible, the closure $\overline{f(Z)}$ must be contained in some irreducible component of $\mathcal{O}(X, Y)$. In particular, if $\mathcal{O}(X, Y)$ is reducible, it is not $\mathbb{D}$-dominated.

Example 2.7. Let $Y$ be a complex space containing a nontrivial entire curve $\mathbb{C} \rightarrow$ $Y$. Assume that there exists a proper closed complex subvariety $Y^{\prime} \subsetneq Y$ which contains all nontrivial entire curves. Then $\mathcal{O}(\mathbb{C}, Y)$ is reducible. Indeed, if we identify the set of constant maps $\mathbb{C} \rightarrow Y$ with $Y$, then $Y$ and $\mathcal{O}\left(\mathbb{C}, Y^{\prime}\right)$ are proper Zariski closed subset of $\mathcal{O}(\mathbb{C}, Y)$ and we have $\mathcal{O}(\mathbb{C}, Y)=Y \cup \mathcal{O}\left(\mathbb{C}, Y^{\prime}\right)$. Thus, we cannot omit the assumption that $\Omega$ is bounded in Theorem 1.2 .

Example 2.8. Let $A=\{z \in \mathbb{C}: 1 / r<|z|<r\}, r>1$ be an annulus. Note that $\mathcal{O}(A, A)$ has three path components, namely the component $\mathcal{P}_{0}$ of null-homotopic maps, the component $\mathcal{P}_{1}$ of degree one maps and the component $\mathcal{P}_{-1}$ of degree minus one maps. Then $\mathcal{P}_{0}$ is $\mathbb{D}$-dominated (it follows from the $\mathbb{D}$-dominability of $\mathcal{O}(A, \mathbb{D})$ ), but others are not $\mathbb{D}$-dominated because $A$ is immobile and Aut $A=$ $\mathcal{P}_{1} \cup \mathcal{P}_{-1}$. It also follows that the Zariski topology on Aut $A$ coincides with the compact-open topology. Thus $\mathcal{P}_{1}$ and $\mathcal{P}_{-1}$ are highly reducible. By this example, we cannot replace the assumption that $\Omega$ is convex by the pseudoconvexity in Theorem 1.2 even if we consider the $\mathbb{D}$-dominability of path components.

Example 2.9. Forstnerič and Winkelmann [6] showed that there exists an irreducible singular surface $Y$ such that the set of dense holomorphic discs $\mathbb{D} \rightarrow Y$ is not dense in $\mathcal{O}(\mathbb{D}, Y)$. By Remark 2.4, $\mathcal{O}(\mathbb{D}, Y)$ is not $\mathbb{D}$-dominated for such a 
surface $Y$. Thus, we cannot omit the assumption that $Y$ is nonsingular in Theorem 1.2 .

\section{The $\mathbb{C}$-dominability and Oka manifolds: Proof of Theorem 1.3}

We briefly review the definition of an Oka manifold and Forstnerič's Oka principle. For further details on Oka theory, we refer to the comprehensive monograph [4] and the survey [5].

Let $z=\left(z_{1}, \ldots, z_{n}\right)$, with $z_{j}=x_{j}+i y_{j}$, denote the complex coordinates on $\mathbb{C}^{n}$. A map from a compact set $K$ is said to be holomorphic if it is holomorphic on an open neighborhood of $K$. We denote by $\mathcal{O}(K, Y)$ the space of holomorphic maps $K \rightarrow Y$ equipped with the compact-open topology.

Definition 3.1. A special convex pair $(K, Q)$ in $\mathbb{C}^{n}$ consists of a closed cube $Q=\left\{z \in \mathbb{C}^{n}:\left|x_{j}\right| \leq a_{j},\left|y_{j}\right| \leq b_{j}, j=1, \ldots, n\right\}$ and a compact convex set $K=\left\{z \in Q: y_{n} \leq h\left(z_{1}, \ldots, z_{n-1}, x_{n}\right)\right\}$, where $h$ is a smooth concave function with values in $\left(-b_{n}, b_{n}\right)$.

A complex manifold $Y$ is called an Oka manifold if for each special convex pair $(K, Q)$ the restriction map $\mathcal{O}(Q, Y) \rightarrow \mathcal{O}(K, Y)$ has dense image.

The most important property of Oka manifolds is that maps from Stein spaces to Oka manifolds enjoy the following Oka principle.

Theorem 3.2 (cf. [4, Theorem 5.4.4]). Assume that $X$ is a Stein space and $Y$ is an Oka manifold. Let $K$ be a compact $\mathcal{O}(X)$-convex subset of $X, X^{\prime}$ a closed complex subvariety of $X, P_{0} \subset P$ compact sets in a Euclidean space $\mathbb{R}^{n}$ and $f: P \times X \rightarrow Y$ a continuous map such that

(a) for any $p \in P, f(p, \cdot): X \rightarrow Y$ is holomorphic on a neighborhood of $K$ (independent of $p$ ) and on $X^{\prime}$, and

(b) $f(p, \cdot)$ is holomorphic on $X$ for all $p \in P_{0}$.

Then there exists a homotopy $f_{t}: P \times X \rightarrow Y, t \in[0,1]$ with $f_{0}=f$ such that each $f_{t}$ satisfies properties (a) and (b), and the following hold:

(i) $f_{1}(p, \cdot)$ is holomorphic on $X$ for all $p \in P$,

(ii) $f_{t}$ is uniformly close to $f$ on $P \times K$ for all $t \in[0,1]$, and

(iii) $f_{t}=f$ on $\left(P_{0} \times X\right) \cup\left(P \times X^{\prime}\right)$ for all $t \in[0,1]$.

Let us prove Theorem 1.3. In fact, we shall prove the following stronger result.

Theorem 3.3. Let $Y$ be a connected complex manifold. Assume that for any bounded convex domain $\Omega \Subset \mathbb{C}^{n}$ and any pair of nonempty open subsets $\mathcal{U}, \mathcal{V} \subset$ $\mathcal{O}(\Omega, Y)$, there exists an entire curve $f: \mathbb{C} \rightarrow \mathcal{O}(\Omega, Y)$ such that $f(0) \in \mathcal{U}$ and $f(1) \in \mathcal{V}$. Then $Y$ is Oka.

Proof. Let $(K, Q)$ be a special convex pair in $\mathbb{C}^{n}$ and write $K=\left\{z \in Q: y_{n} \leq\right.$ $\left.h\left(z_{1}, \ldots, z_{n-1}, x_{n}\right)\right\}$ as in Definition 3.1. We would like to show that the restriction map $\mathcal{O}(Q, Y) \rightarrow \mathcal{O}(K, Y)$ has dense image. 
Take an arbitrary open neighborhood $W$ of $K$. By considering tangent spaces of the graph of the smooth concave function $h$, we may find $\mathbb{R}$-affine functions $h_{1}, \ldots, h_{k}$ on $\mathbb{C}^{n-1} \times \mathbb{R} \cong \mathbb{R}^{2 n-1}$ such that

$$
K \subset\left\{z \in Q: y_{n} \leq h_{j}\left(z_{1}, \ldots, z_{n-1}, x_{n}\right), j=1, \ldots, k\right\} \subset W .
$$

For each $l=1, \ldots, k$, let $K_{l}=\left\{z \in Q: y_{n} \leq h_{j}\left(z_{1}, \ldots, z_{n-1}, x_{n}\right), j=1, \ldots, l\right\}$ and $K_{0}=Q$. In order to approximate a holomorphic map in $\mathcal{O}(K, Y)$ which is holomorphic on $W$, it suffices to show that each restriction map $\mathcal{O}\left(K_{l-1}, Y\right) \rightarrow$ $\mathcal{O}\left(K_{l}, Y\right)$ has dense image for $l=1, \ldots, k$. For this purpose, we use a gluing method in Oka theory (cf. [4, §5.9]).

Let $g_{0}: \Omega \rightarrow Y$ be a holomorphic map from an open neighborhood of $K_{l}$. We may assume that $\Omega$ is a bounded convex domain. Let us construct a smooth strongly pseudoconvex Cartan pair $\left(D_{0}, D_{1}\right)$ (for the notion of a Cartan pair, see [4, Definition 5.7.1]) such that if we set $L=\overline{D_{0} \cap D_{1}}$ then

(a) $K_{l} \subset D_{0} \Subset \Omega, K_{l-1} \subset D_{0} \cup D_{1}$,

(b) $K_{l} \cap \widehat{L}_{\mathcal{O}\left(\mathbb{C}^{n}\right)}=\emptyset$, and $K_{l} \cup \widehat{L}_{\mathcal{O}\left(\mathbb{C}^{n}\right)}$ is polynomially convex.

Choose a small number $\varepsilon>0$ such that $A=\left\{z \in K_{l-1}: y_{n} \leq h_{l}\left(z_{1}, \ldots, z_{n-1}, x_{n}\right)+\right.$ $3 \varepsilon\} \subset \Omega$ and let $B=\left\{z \in K_{l-1}: y_{n} \geq h_{l}\left(z_{1}, \ldots, z_{n-1}, x_{n}\right)+2 \varepsilon\right\}$. Then $(A, B)$ is a Cartan pair in $\mathbb{C}^{n}$ because $A, B, A \cup B$ and $A \cap B$ are convex and $\overline{A \backslash B} \cap \overline{B \backslash A}=\emptyset$. Note that $K_{l} \subset A$ and $K_{l-1}=A \cup B$. For the open neighborhood $\Omega$ of $A$ and the open neighborhood $U=\left\{z \in \mathbb{C}^{n}: y_{n}>h_{k}\left(z_{1}, \ldots, z_{n-1}, x_{n}\right)+\varepsilon\right\}$ of $B$, there exists a smooth strongly pseudoconvex Cartan pair $\left(D_{0}, D_{1}\right)$ such that $A \subset D_{0} \Subset \Omega$ and $B \subset D_{1} \Subset U$ (see [4, Proposition 5.7.3]). Then $\left(D_{0}, D_{1}\right)$ satisfies the required properties (see Proposition 4.2).

Put $m=\operatorname{dim} Y$. The composition of the projection map $\bar{D}_{1} \times \mathbb{B}^{m} \rightarrow \mathbb{B}^{m}$ and an open embedding map $\mathbb{B}^{m} \rightarrow Y$ gives a dominating local holomorphic spray $\pi: \bar{D}_{1} \times \mathbb{B}^{m} \rightarrow Y$ (for the notion of a local holomorphic spray, see [4, Definition 5.9.1]).

By assumption there exists an entire curve $f: \mathbb{C} \rightarrow \mathcal{O}\left(\Omega \times \mathbb{B}^{m}, Y\right)$ such that $f(0)(\cdot, 0)$ and $f(1)$ approximate sufficiently $g_{0}$ on $K_{l}$ and $\pi$ on $L \times \mathbb{B}^{m}$, respectively. By the conditions (a) and (b) of $\left(D_{0}, D_{1}\right)$ and by Oka-Weil theorem, there exists $\varphi \in \mathcal{O}\left(\mathbb{C}^{n+m}\right)$ such that $\left.\varphi\right|_{K_{l} \times \mathbb{B}^{m}}$ is sufficiently close to 0 and $\left.\varphi\right|_{L \times \mathbb{B}^{m}}$ is sufficiently close to 1 . Consider $g=\operatorname{ev}\left(f \circ \varphi, \operatorname{id}_{\bar{D}_{0} \times \mathbb{B}^{m}}\right): \bar{D}_{0} \times \mathbb{B}^{m} \rightarrow Y$. It is a local holomorphic spray which is sufficiently close to $\pi$ on $L \times \mathbb{B}^{m}$ and $g(\cdot, 0)$ is sufficiently close to $g_{0}$ on $K_{l}$. The closeness between $g$ and $\pi$ on $L \times \mathbb{B}^{m}$ implies that there exist a small number $0<\delta<1$ and a continuous map $\gamma: L \times \delta \mathbb{B}^{m} \rightarrow L \times \mathbb{B}^{m}$ of the form $\gamma(z, w)=(z, \psi(z, w))$ which is holomorphic on $\left(D_{0} \cap D_{1}\right) \times \delta \mathbb{B}^{m}$, sufficiently close to the inclusion map $L \times \delta \mathbb{B}^{m} \hookrightarrow L \times \mathbb{B}^{m}$ and satisfies $g \circ \gamma=\pi$ on $L \times \delta \mathbb{B}^{m}$ (see [4, Lemma 5.9.3]). Then the closeness between $\gamma$ and the inclusion map implies that there exist holomorphic maps

$$
\alpha: D_{0} \times \delta^{2} \mathbb{B}^{m} \rightarrow D_{0} \times \delta \mathbb{B}^{m}, \beta: D_{1} \times \delta^{2} \mathbb{B}^{m} \rightarrow D_{1} \times \delta \mathbb{B}^{m}
$$


which are sufficiently close to the inclusion maps and satisfy $\gamma \circ \beta=\alpha$ on $\left(D_{0} \cap\right.$ $\left.D_{1}\right) \times \delta^{2} \mathbb{B}^{m}$ (see [4, Proposition 5.8.1]). Since $g \circ \alpha=\pi \circ \beta$ on $\left(D_{0} \cap D_{1}\right) \times \delta^{2} \mathbb{B}^{m}$, $g \circ \alpha$ and $\pi \circ \beta$ amalgamate into a holomophic map $g^{\prime}:\left(D_{0} \cup D_{1}\right) \times \delta^{2} \mathbb{B}^{m} \rightarrow Y$ such that the restricted map $g^{\prime}(\cdot, 0): K_{l-1} \rightarrow Y$ approximates $g_{0}$ on $K_{l}$.

Proof of Theorem 1.3. (1) $\Longrightarrow(2)$ : It is an easy application of Oka principle (Theorem 3.2). We may choose a dense sequence $\left\{f_{j}\right\}_{j \in \mathbb{N}} \subset \mathcal{P}$ since $\mathcal{P}$ is separable. Since there exists a continuous map $f: \mathbb{C} \times X \rightarrow Y$ such that $f(j, \cdot)=f_{j}$ for all $j \in \mathbb{N}$, there exists a holomorphic map $\tilde{f}: \mathbb{C} \times X \rightarrow Y$ satisfying the same condition by Theorem [3.2. The associated map to $\tilde{f}$ gives a dense entire curve $\mathbb{C} \rightarrow \mathcal{P}$.

$(2) \Longrightarrow(3)$ : It is obvious since bounded convex domains are Stein.

$(3) \Longrightarrow(1)$ : Since a dense entire curve in $\mathcal{O}(\Omega, Y)$ intersects all nonempty open subsets in $\mathcal{O}(\Omega, Y)$, Theorem 3.3 implies that $Y$ is Oka.

We explain another characterization of Oka manifolds which can be obtained by Theorem 3.3. Let $X$ and $Y$ be complex spaces. We say a subspace $\mathcal{S} \subset \mathcal{O}(X, Y)$ to be strongly $\mathbb{C}$-connected if any pair of points in $\mathcal{S}$ can be joined by an entire curve $\mathbb{C} \rightarrow \mathcal{S}$. Note that for any Stein space $X$ and any Oka manifold $Y$, every path component of $\mathcal{O}(X, Y)$ is strongly $\mathbb{C}$-connected by Theorem 3.2 . The following characterization of Oka manifolds is also an immediate corollary of Theorem 3.3 .

Corollary 3.4. For a connected complex manifold $Y$, the following are equivalent:

(1) $Y$ is Oka.

(2) For any Stein space $X$, every path component $\mathcal{P} \subset \mathcal{O}(X, Y)$ is strongly $\mathbb{C}$-connected.

(3) For any bounded convex domain $\Omega \Subset \mathbb{C}^{n}$, the space $\mathcal{O}(\Omega, Y)$ is strongly $\mathbb{C}$-connected.

The above characterization can be reformulated into more interesting form. For $x \in X$, we write $\mathrm{ev}_{x}=\mathrm{ev}(\cdot, x): \mathcal{O}(X, Y) \rightarrow Y$. As we mentioned in Remark 1.8, for any holomorphic map $f: Z \rightarrow \mathcal{O}(X, Y)$ from a complex space, the composition $\mathrm{ev}_{x} \circ f: Z \rightarrow Y$ is holomorphic. Therefore it is interesting to ask when $\left(\mathrm{ev}_{0}, \mathrm{ev}_{1}\right)$ : $\mathcal{O}(\mathbb{C}, Y) \rightarrow Y^{2}$ is an Oka map, namely we call it an Oka map if it is a Serre fibration and it enjoys the parametric Oka property for Euclidean parameter spaces (in the obvious sense, see [4, Definition 6.14.7]).

Corollary 3.5. For a connected complex manifold $Y$, the following are equivalent:

(1) $Y$ is Oka.

(2) $\left(\mathrm{ev}_{0}, \mathrm{ev}_{1}\right): \mathcal{O}(\mathbb{C}, Y) \rightarrow Y^{2}$ is an Oka map.

(3) For any bounded convex domain $\Omega \Subset \mathbb{C}^{n}$, every holomorphic map $\Omega \rightarrow Y^{2}$ lifts to a holomorphic map $\Omega \rightarrow \mathcal{O}(\mathbb{C}, Y)$.

Proof. The condition (3) is a simple rephrasing of the condition (3) in Corollary 3.4. We only need to prove that $(1) \Longrightarrow(2)$. Assume that $Y$ is Oka and denote 
by $\iota:\{0,1\} \hookrightarrow \mathbb{C}$ the inclusion map. Note that $\left(\mathrm{ev}_{0}, \mathrm{ev}_{1}\right): \mathcal{O}(\mathbb{C}, Y) \rightarrow Y^{2}$ can be identified with the precomposition map $\iota^{*}: \mathcal{O}(\mathbb{C}, Y) \rightarrow \mathcal{O}(\{0,1\}, Y)$. Therefore $\left(\mathrm{ev}_{0}, \mathrm{ev}_{1}\right)$ is a Serre fibration (see [9, §16]). By considering the associated map to $P \times X \rightarrow \mathcal{O}(\mathbb{C}, Y)$ and using the parametric Oka property of $Y$ (see Theorem 3.2), we may easily obtain the parametric Oka property of $\left(\mathrm{ev}_{0}, \mathrm{ev}_{1}\right)$.

\section{UNIVERSAl MAPs: APPliCATIONS OF DENSE hOLOMORPHIC CURVES}

The following characterizes automorphisms admitting universal functions.

Theorem 4.1 (cf. [11, Theorem 6]). Let $X$ be a Stein space and $\tau \in$ Aut $X$ an automorphism. Then there exists an $\mathcal{O}(X)$-universal function for $\tau$ if and only if for any compact $\mathcal{O}(X)$-convex subset $K \subset X$ there exists $j \in \mathbb{N}$ such that $\tau^{j}(K) \cap K=\emptyset$ and $\tau^{j}(K) \cup K$ is $\mathcal{O}(X)$-convex.

For a connected Stein manifold, the above theorem is due to Zając [11]. For arbitrary Stein space, this follows immediately from the following generalization of Lemma 2 in [11] to a Stein space (see the proof of Theorem 6 in [11]). We may prove the following by choosing a relatively compact open Runge neighborhood of $\widehat{K}_{\mathcal{O}(X)} \cup \widehat{L}_{\mathcal{O}(X)}$ and embedding it to a Euclidean space, but we omit the details (see the proof of Lemma 2 in [11]).

Proposition 4.2 (cf. [11, Lemma 2]). Let $X$ be a Stein space. For compact subsets $K, L \subset X$, the following are equivalent:

(1) There exists $f \in \mathcal{O}(X)$ such that $\widehat{f(K)}_{\mathcal{O}(\mathbb{C})} \cap \widehat{f(L)}_{\mathcal{O}(\mathbb{C})}=\emptyset$.

(2) $\widehat{K}_{\mathcal{O}(X)} \cap \widehat{L}_{\mathcal{O}(X)}=\emptyset$ and $(\widehat{K \cup L})_{\mathcal{O}(X)}=\widehat{K}_{\mathcal{O}(X)} \cup \widehat{L}_{\mathcal{O}(X)}$.

In particular, if $K$ and $L$ are disjoint and $\mathcal{O}(X)$-convex, then $K \cup L$ is $\mathcal{O}(X)$-convex if and only if $K$ and $L$ satisfy the condition (1).

Following Theorem 4.1, we give the definition of a generalized translation.

Definition 4.3. Let $X$ be a Stein space. We call $\tau \in$ Aut $X$ a generalized translation if for any compact $\mathcal{O}(X)$-convex subset $K \subset X$ there exists $j \in \mathbb{N}$ such that

(1) $\tau^{j}(K) \cap K=\emptyset$, and

(2) $\tau^{j}(K) \cup K$ is $\mathcal{O}(X)$-convex.

The following proposition gives a lot of examples of generalized translations.

Proposition 4.4. Let $S$ and $X$ be Stein spaces and $f: S \rightarrow X$ a holomorphic map. Assume that $\tau \in$ Aut $X$ is a generalized translation and $\sigma \in$ Aut $S$ is an automorphism such that $\tau \circ f=f \circ \sigma$. Then $\sigma$ is also a generalized translation.

Proof. Let $K \subset S$ be a compact $\mathcal{O}(S)$-convex subset and let $L=\widehat{f(K)}_{\mathcal{O}(X)}$. Since $\tau$ is a generalized translation, there exists $j \in \mathbb{N}$ such that $\tau^{j}(L) \cap L=\emptyset$ and $\tau^{j}(L) \cup L$ is $\mathcal{O}(X)$-convex. By assumption, it follows that $f\left(\sigma^{j}(K)\right) \cap f(K)=\emptyset$. 
Thus $\sigma^{j}(K) \cap K=\emptyset$. The $\mathcal{O}(S)$-convexity of $\sigma^{j}(K) \cup K$ follows easily from Proposition 4.2.

We may obtain the following corollary by considering the projection map and the inclusion map.

Corollary 4.5. Let $X$ be a Stein space and $\tau \in$ Aut $X$ a generalized translation.

(1) If $Y$ is a Stein space and $\sigma \in \operatorname{Aut}(X \times Y)$ is of the form $\sigma(x, y)=$ $(\tau(x), f(x, y))$, then $\sigma$ is a generalized translation.

(2) If $\Omega \subset X$ is a Stein subset such that $\tau(\Omega)=\Omega$, then $\left.\tau\right|_{\Omega} \in$ Aut $\Omega$ is a generalized translation.

Example 4.6. A usual translation of $\mathbb{C}^{n}$ is clearly a generalized translation. By Proposition 4.7 , a ball $\mathbb{B}^{n}$ and a polydisc $\mathbb{D}^{n}$ have generalized translations (it also follows from Corollary 4.5 and the Cayley transform $\mathbb{B}^{n} \cong\left\{\left(z^{\prime}, z_{n}\right) \in \mathbb{C}^{n}: \mathfrak{I} z_{n}>\right.$ $\left.\left\|z^{\prime}\right\|^{2}\right\}$ ). Therefore, for any Stein space $X$, the product spaces $\mathbb{C} \times X$ and $\mathbb{D} \times X$ have generalized translations of the form $\tau=\sigma \times \mathrm{id}_{X}$ by Corollary 4.5.

4.1. Universal maps from bounded convex domains. The following gives a simple characterization of generalized translations on bounded convex domains. Recall that $\left\{\varphi_{j}\right\}_{j \in \mathbb{N}} \subset$ Aut $\Omega$ is said to be compactly divergent if for any compact subset $K \subset X$ there exists $N \in \mathbb{N}$ such that $\varphi_{j}(K) \cap K=\emptyset$ for all $j \geq N$.

Proposition 4.7. Let $\Omega \Subset \mathbb{C}^{n}$ be a bounded convex domain. For an automorphism $\tau \in$ Aut $\Omega$, the following are equivalent:

(1) $\tau$ is a generalized translation.

(2) $\tau$ has no fixed points.

(3) $\left\{\tau^{j}\right\}_{j \in \mathbb{N}}$ is compactly divergent.

Proof. It is obvious that $(1) \Longrightarrow(2)$. Since $\Omega$ is a bounded convex domain, $(2) \Longleftrightarrow(3)$ (cf. [1, Theorem 2.4.20]). The implication (3) $\Longrightarrow$ (1) follows from Theorem 1.2 and the proof of Corollary 4.9 below (see also [11, Theorem 11]).

To prove Theorem 1.5, we shall need the following lemma. A complex space $X$ is said to be $c$-finitely compact if $X$ is Carathéodory hyperbolic and every closed ball (with respect to the Carathéodory distance $c_{X}$ ) in $X$ is compact. It is known that a $c$-finitely compact space is Stein (cf. [8, Corollary 4.4.7]).

Lemma 4.8. Let $X$ be a c-finitely compact space, $\Omega \Subset \mathbb{C}^{n}$ a bounded convex domain and $\tau \in$ Aut $X$ be an automorphism such that $\left\{\tau^{j}\right\}_{j \in \mathbb{N}}$ is compactly divergent. Then there exists an $\mathcal{O}(X, \Omega)$-universal map for $\tau$.

Proof. By Birkhoff's transitivity theorem (cf. [7, Theorem 1.16]), it suffices to show that for any pair of nonempty open subsets $\mathcal{U}, \mathcal{V} \subset \mathcal{O}(X, \Omega)$ there exists $j \in \mathbb{N}$ such that $\left(\tau^{*}\right)^{j}(\mathcal{U}) \cap \mathcal{V} \neq \emptyset$. Take $u \in \mathcal{U}, v \in \mathcal{V}$ with relatively compact images $u(X), v(X) \Subset \Omega$. Since $\Omega$ is taut, there exists a subsequence $\left\{\tau^{j_{k}}\right\}_{k \in \mathbb{N}}$ of $\left\{\tau^{j}\right\}$ such that $\left\{u \circ \tau^{j_{k}}\right\}_{k \in \mathbb{N}}$ and $\left\{v \circ \tau^{-j_{k}}\right\}_{k \in \mathbb{N}}$ have limits $\widetilde{u}, \widetilde{v} \in \mathcal{O}(X, \Omega)$, respectively. 
For $j=1,2$, we denote by $\pi_{j}$ the projection map $\Omega^{2} \rightarrow \Omega,\left(x_{1}, x_{2}\right) \mapsto x_{j}$. Since $\pi_{1}(u, \widetilde{v})=u \in \mathcal{U}, \pi_{2}(\widetilde{u}, v)=v \in \mathcal{V}$, there exists a holomorphic map $f: \overline{\mathbb{D}} \rightarrow$ $\mathcal{O}\left(\Omega^{2}, \Omega\right)$ such that $f(1) \circ(u, \widetilde{v}) \in \mathcal{U}$ and $f(-1) \circ(\widetilde{u}, v) \in \mathcal{V}$ by Theorem 1.2 .

Take $x \in X$. Since $\left\{\tau^{j_{k}}\right\}_{k \in \mathbb{N}}$ is compactly divergent and $X$ is $c$-finitely compact, $c_{X}\left(x, \tau^{j_{k}}(x)\right) \rightarrow \infty$ as $k \rightarrow \infty$. Thus there exists a sequence $\left\{\varphi_{k}: \Omega \rightarrow \mathbb{D}\right\}_{k \in \mathbb{N}}$ of holomorphic maps such that $\varphi_{k}(x) \rightarrow-1$ and $\varphi_{k} \circ \tau^{j_{k}}(x) \rightarrow 1$. By Montel's theorem and the maximum principle, passing to a subsequence if necessary, we may assume that $\varphi_{k} \rightarrow-1$ and $\varphi_{k} \circ \tau^{j_{k}} \rightarrow 1$.

For each $k \in \mathbb{N}$, let $F_{k}=\operatorname{ev}\left(f \circ \varphi_{k},\left(u, v \circ \tau^{-j_{k}}\right)\right) \in \mathcal{O}(X, \Omega)$. By our construction, $F_{k} \rightarrow f(1) \circ(u, \widetilde{v}) \in \mathcal{U}$ and $F_{k} \circ \tau^{j_{k}} \rightarrow f(-1) \circ(\widetilde{u}, v) \in \mathcal{V}$ as $k \rightarrow \infty$. Thus it follows that $\left(\tau^{*}\right)^{j_{k}}(\mathcal{U}) \cap \mathcal{V} \neq \emptyset$ for any sufficiently large $k \in \mathbb{N}$.

The following corollary and Theorem 1.2 imply Theorem 1.5 .

Corollary 4.9. Let $\Omega \Subset \mathbb{C}^{n}$ be a bounded convex domain, $\tau \in$ Aut $\Omega$ a generalized translation and $Y$ a complex space. Let $\mathcal{Z} \subset \mathcal{O}(\Omega, Y)$ be a $\tau^{*}$-invariant irreducible component (with respect to the Zariski topology, see Definition 2.6). Then $\mathcal{Z}$ is $\mathbb{D}$-dominated if and only if there exists a $\mathcal{Z}$-universal map for $\tau$.

Proof. Let us assume that $\mathcal{Z}$ is $\mathbb{D}$-dominated, i.e. there exists a dense holomorphic $\operatorname{disc} f: \mathbb{D} \rightarrow \mathcal{Z}$. Let $\widehat{f}: \mathbb{D} \times \Omega \rightarrow Y$ be the associated holomorphic map. Since $\mathcal{O}(\Omega, \mathbb{D} \times \Omega)$ is irreducible (it follows from Theorem 1.2), it defines a dense holomorphic map $\widehat{f}_{*}: \mathcal{O}(\Omega, \mathbb{D} \times \Omega) \rightarrow \mathcal{Z}$. Since the bounded convex domain $\Omega$ is $c$-finitely compact (cf. [8, Corollary 4.1.10]), there exists an $\mathcal{O}(\Omega, \mathbb{D} \times \Omega$ )-universal map $F$ by Lemma 4.8. Then its image $\widehat{f}_{*}(F)=F \circ f$ is a $\mathcal{Z}$-universal map since $\tau^{*} \circ \widehat{f}_{*}=\widehat{f}_{*} \circ \tau^{*}$.

If there exists a $\mathcal{Z}$-universal map $F: \Omega \rightarrow Y$, then a dense holomorphic disc $f$ : $\mathbb{D} \rightarrow \mathcal{O}(\Omega, \Omega)$ (see Theorem 1.2) and the postcomposition map $F_{*}: \mathcal{O}(\Omega, \Omega) \rightarrow \mathcal{Z}$ give a dense holomorphic disc $F_{*} \circ f: \mathbb{D} \rightarrow \mathcal{Z}$.

Remark 4.10. From the proof of Lemma 4.8, in the situation of Theorem 1.5, it also follows that the precomposition map $\tau^{*}: \mathcal{O}(\Omega, Y) \rightarrow \mathcal{O}(\Omega, Y)$ is hereditarily hypercyclic, i.e. for any subsequence $\left\{\tau^{j_{k}}\right\}_{k \in \mathbb{N}}$ of $\left\{\tau^{j}\right\}_{j \in \mathbb{N}}$ there exists $F \in \mathcal{O}(\Omega, Y)$ such that $\left\{F \circ \tau^{j_{k}}\right\}_{k \in \mathbb{N}}$ is dense in $\mathcal{O}(\Omega, Y)$ (see also [11, Theorem 14]).

As an application, we may obtain the following stronger version of Theorem 3.3 .

Corollary 4.11. Let $Y$ be a connected complex manifold. Assume that for any bounded convex domain $\Omega \Subset \mathbb{C}^{n}$ there exists a nonempty open subset $\mathcal{W} \subset \mathcal{O}(\Omega, Y)$ satisfying the following property: for any pair of nonempty open subsets $\mathcal{U}, \mathcal{V} \subset W$ there exists an entire curve $f: \mathbb{C} \rightarrow \mathcal{O}(\Omega, Y)$ such that $f(0) \in \mathcal{U}, f(1) \in \mathcal{V}$. Then $Y$ is Oka.

Proof. We verify the condition in Theorem 3.3. Let $\Omega \Subset \mathbb{C}^{n}$ be a bounded convex domain and $\mathcal{U}, \mathcal{V}$ be a pair of nonempty open subsets of $\mathcal{O}(\Omega, Y)$. The inclusion 
map $\iota: \Omega \hookrightarrow \mathbb{D} \times \Omega, x \mapsto(0, x)$ induces the surjective precomposition map $\iota^{*}: \mathcal{O}(\mathbb{D} \times \Omega, Y) \rightarrow \mathcal{O}(\Omega, Y)$. We write $\mathcal{U}^{\prime}=\left(\iota^{*}\right)^{-1}(\mathcal{U})$ and $\mathcal{V}^{\prime}=\left(\iota^{*}\right)^{-1}(\mathcal{V})$

Note that $\mathbb{D} \times \Omega$ has a generalized translation $\tau \in \operatorname{Aut}(\mathbb{D} \times \Omega)$. Let $\mathcal{W} \subset$ $\mathcal{O}(\mathbb{D} \times \Omega, Y)$ be an open set as in the assumption. By the hereditary hypercyclicity of $\tau^{*}: \mathcal{O}(\mathbb{D} \times \Omega, Y) \rightarrow \mathcal{O}(\mathbb{D} \times \Omega, Y)$ (see Remark 4.10), we may find $j \in \mathbb{N}$ such that $\left(\tau^{*}\right)^{j}(\mathcal{W}) \cap \mathcal{U}^{\prime} \neq \emptyset$ and $\left(\tau^{*}\right)^{j}(\mathcal{W}) \cap \mathcal{V}^{\prime} \neq \emptyset$. By assumption, there exists an entire curve $f: \mathbb{C} \rightarrow \mathcal{O}(\Omega, Y)$ such that $f(0) \circ \tau^{j} \in \mathcal{U}^{\prime}$ and $f(1) \circ \tau^{j} \in \mathcal{V}^{\prime}$. Then the entire curve $\widetilde{f}=\left(\tau^{j} \circ \iota\right)^{*} \circ f: \mathbb{C} \rightarrow \mathcal{O}(\Omega, Y)$ satisfies $\tilde{f}(0) \in \mathcal{U}$ and $\widetilde{f}(1) \in \mathcal{V}$.

From the above corollary, it follows that a connected complex manifold $Y$ is Oka if and only if for any bounded convex domain $\Omega \Subset \mathbb{C}^{n}$ there exists a somewhere dense entire curve $\mathbb{C} \rightarrow \mathcal{O}(\Omega, Y)$ (compare with Theorem 1.3).

4.2. Universal maps from general Stein spaces. We shall show that the $\mathbb{C}$ dominability implies the existence of a universal map from a more general Stein space than bounded convex domains. Theorem 4.12 and Corollary 4.13 are stated for path components, but the analogous ones hold for irreducible components. At present, the author does not know whether irreducible components are pathconnected.

Theorem 4.12. Let $X$ be a Stein space, $\tau \in$ Aut $X$ a generalized translation and $Y$ a complex space. Let $\mathcal{P}$ be a $\tau^{*}$-invariant path component of $\mathcal{O}(X, Y)$ and assume that $\mathcal{P}$ is $\mathbb{C}$-dominated. Then there exists a $\mathcal{P}$-universal map for $\tau$.

Proof. Let $f: \mathbb{C} \rightarrow \mathcal{P}$ be a dense entire curve. Choose a countable base $\left\{\mathcal{U}_{j}\right\}_{j \in \mathbb{N}}$ for the topology of $\mathcal{P}$ and a distance function $d$ of $X$. Assume that $\mathcal{U}_{j} \neq \emptyset$ for all $j \in \mathbb{N}$. For each $j \in \mathbb{N}$, there exist a compact subset $K_{j} \subset X$, a holomorphic map $h_{j} \in \mathcal{P}$ and $\varepsilon_{j}>0$ such that $\left\{g \in \mathcal{P}: \sup _{K_{j}} d\left(g, h_{j}\right)<\varepsilon_{j}\right\} \subset \mathcal{U}_{j}$. For each $j, k \in \mathbb{N}$, we denote by $\mathcal{V}_{j, k}$ the open set of holomorphic functions $g \in \mathcal{O}(X)$ satisfying $\operatorname{ev}\left(f \circ g, \operatorname{id}_{X}\right) \in\left(\tau^{*}\right)^{-k}\left(\mathcal{U}_{j}\right)$. Note that, since $f: \mathbb{C} \rightarrow \mathcal{P}$ has dense image, for each $j, k \in \mathbb{N}$ there exist $a_{j, k} \in \mathbb{C}$ and $\varepsilon_{j, k}>0$ such that $\{g \in \mathcal{O}(X)$ : $\left.\sup _{\tau^{k}\left(K_{j}\right)}\left|g-a_{j, k}\right|<\varepsilon_{j, k}\right\} \subset \mathcal{V}_{j, k}$.

Let us show that $\bigcup_{k \in \mathbb{N}} \mathcal{V}_{j, k}$ is dense in $\mathcal{O}(X)$ for each $j \in \mathbb{N}$. Take a holomorphic function $h \in \mathcal{O}(X)$, a compact $\mathcal{O}(X)$-convex subset $L \supset K_{j}$ of $X$ and $\varepsilon>0$. By definition, there exists $k \in \mathbb{N}$ such that $\tau^{k}(L) \cap L=\emptyset$ and $\tau^{k}(L) \cup L$ is $\mathcal{O}(X)$ convex. By Oka-Weil theorem, there exists a holomorphic function $g \in \mathcal{O}(X)$ such that

(a) $\sup _{L}|g-h|<\varepsilon$, and

(b) $\sup _{\tau^{k}\left(K_{j}\right)}\left|g-a_{j, k}\right|<\varepsilon_{j, k}$.

This proves the density of $\bigcup_{k \in \mathbb{N}} \mathcal{V}_{j, k}$. It follows that $\bigcap_{j \in \mathbb{N}} \bigcup_{k \in \mathbb{N}} \mathcal{V}_{j, k} \neq \emptyset$ since $\mathcal{O}(X)$ is a Baire space. This means that there exists a holomorphic function $\varphi \in \mathcal{O}(X)$ such that for each $j \in \mathbb{N}$ there exists $k \in \mathbb{N}$ such that $\operatorname{ev}\left(f \circ \varphi \circ \tau^{k}, \tau^{k}\right) \in \mathcal{U}_{j}$. Then $\operatorname{ev}\left(f \circ \varphi, \operatorname{id}_{X}\right) \in \mathcal{P}$ is a $\mathcal{P}$-universal map for $\tau$. 
By Theorem 1.3 and Theorem 4.12, we obtain the implication (1) $\Longrightarrow(2)$ in Theorem 1.6. Let us prove the remaining implication $(3) \Longrightarrow(1)$.

Proof of Theorem 1.6. Let $\Omega \Subset \mathbb{C}^{n}$ be a bounded convex domain. There exists a generalized translation $\tau \in \operatorname{Aut}(\mathbb{C} \times \Omega)$ of the form $\tau=\sigma \times \operatorname{id}_{\Omega}$ (see Example 4.6). By assumption, there exists a universal map $F: \mathbb{C} \times \Omega \rightarrow Y$ for $\tau$. Note that there exists an entire curve $f: \mathbb{C} \rightarrow \mathcal{O}(\mathbb{C} \times \Omega, \mathbb{C} \times \Omega)$ such that $\left\{\left(\tau \times \operatorname{id}_{\Omega}\right)^{j}\right\}_{j \in \mathbb{N}} \subset \overline{f(\mathbb{C})}$. Using the postcomposition map $F_{*}: \mathcal{O}(\mathbb{C} \times \Omega, \mathbb{C} \times \Omega) \rightarrow \mathcal{O}(\mathbb{C} \times \Omega, Y)$, we obtain a dense entire curve $F_{*} \circ f: \mathbb{C} \rightarrow \mathcal{O}(\mathbb{C} \times \Omega, Y)$. Note that this also gives a dense entire curve $\mathbb{C} \rightarrow \mathcal{O}(\Omega, Y)$. Thus $Y$ is Oka by Theorem 1.3.

A Stein manifold $X$ is called an elliptic Stein manifold if it is also Oka, because it is equivalent to Gromov's ellipticity (see [4, Proposition 5.15.2]). The following corollary is an analogue of Corollary 4.9 for the $\mathbb{C}$-dominability.

Corollary 4.13. Let $X$ be an elliptic Stein manifold, $\tau \in$ Aut $X$ a generalized translation and $Y$ a complex space. Let $\mathcal{P}$ be a $\tau^{*}$-invariant path component of $\mathcal{O}(X, Y)$ and assume that $\tau$ is homotopic to the identity map $\operatorname{id}_{X}$. Then $\mathcal{P}$ is $\mathbb{C}$-dominated if and only if there exists a $\mathcal{P}$-universal map for $\tau$.

Proof. We only need to prove the "if" part. By Theorem $3.2, \tau$ lies in the path component $\mathcal{P} \subset \mathcal{O}(X, X)$ containing id ${ }_{X}$. Thus $\left\{\tau^{j}\right\}_{j \in \mathbb{N}} \subset \mathcal{P}$. By Theorem 1.3, $\mathcal{P}$ is $\mathbb{C}$-dominated. The rest of the proof goes through just as in Corollary 4.9,

\section{ACKNOWLEDGEMENT}

I would like to thank my supervisor Katsutoshi Yamanoi for his interest and encouragement.

\section{REFERENCES}

[1] M. Abate, Iteration theory of holomorphic maps on taut manifolds, Research and Lecture Notes in Mathematics. Complex Analysis and Geometry, Mediterranean Press, Rende, 1989.

[2] R. B. Andrist and E. F. Wold, Free dense subgroups of holomorphic automorphisms, Math. Z. 280 (2015), no. 1-2, 335-346.

[3] B.-Y. Chen and X. Wang, Holomorphic maps with large images, J. Geom. Anal. 25 (2015), no. $3,1520-1546$.

[4] F. Forstnerič, Stein manifolds and holomorphic mappings, Ergebnisse der Mathematik und ihrer Grenzgebiete. 3. Folge. A Series of Modern Surveys in Mathematics [Results in Mathematics and Related Areas. 3rd Series. A Series of Modern Surveys in Mathematics], vol. 56, Springer, Heidelberg, 2011.

[5] _ Oka manifolds: from Oka to Stein and back, Ann. Fac. Sci. Toulouse Math. (6) 22 (2013), no. 4, 747-809.

[6] F. Forstnerič and J. Winkelmann, Holomorphic discs with dense images, Math. Res. Lett. 12 (2005), no. 2-3, 265-268.

[7] K.-G. Grosse-Erdmann and A. Peris, Linear chaos, Universitext, Springer, London, 2011.

[8] S. Kobayashi, Hyperbolic complex spaces, Grundlehren der Mathematischen Wissenschaften [Fundamental Principles of Mathematical Sciences], vol. 318, Springer-Verlag, Berlin, 1998. 
[9] F. Lárusson, Model structures and the Oka principle, J. Pure Appl. Algebra 192 (2004), no. 1-3, 203-223.

[10] J. Winkelmann, Non-degenerate maps and sets, Math. Z. 249 (2005), no. 4, 783-795.

[11] S. Zając, Hypercyclicity of composition operators in Stein manifolds, Proc. Amer. Math. Soc. 144 (2016), no. 9, 3991-4000.

Department of Mathematics, Graduate School of Science, Osaka University, TOYONAKA, OSAKA 560-0043, JAPAN

E-mail address: y-kusakabe@cr.math.sci.osaka-u.ac.jp 